\title{
The PAS/LOV protein VIVID supports a rapidly dampened daytime oscillator that facilitates entrainment of the Neurospora circadian clock
}

\author{
Mark Elvin, ${ }^{1}$ Jennifer J. Loros, ${ }^{2}$ Jay C. Dunlap, ${ }^{2}$ and Christian Heintzen ${ }^{1,3}$ \\ ${ }^{1}$ Faculty of Life Sciences, The University of Manchester, Manchester, M13 9PT, United Kingdom; ${ }^{2}$ Departments of Genetics \\ and Biochemistry, Dartmouth Medical School, Hanover, New Hampshire 03755, USA
}

\begin{abstract}
A light-entrainable circadian clock controls development and physiology in Neurospora crassa. Existing simple models for resetting based on light pulses (so-called nonparametric entrainment) predict that constant light should quickly send the clock to an arrhythmic state; however, such a clock would be of little use to an organism in changing photoperiods in the wild, and we confirm that true, albeit dampened, rhythmicity can be observed in extended light. This rhythmicity requires the PAS/LOV protein VIVID (VVD) that acts, in the light, to facilitate expression of an oscillator that is related to, but distinguishable from, the classic FREQUENCY/WHITE-COLLAR complex (FRQ/WCC)-based oscillator that runs in darkness. VVD prevents light resetting of the clock at dawn but, by influencing frq RNA turnover, promotes resetting at dusk, thereby allowing the clock to run through the dawn transition and take its phase cues from dusk. Consistent with this, loss of VVD yields a clock whose performance follows the simple predictions of earlier models, and overexpression of VVD restores rhythmicity in the light and sensitivity of phase to the duration of the photoperiod.
\end{abstract}

[Keywords: Circadian clock; vivid; white-collar; frequency; limit cycle; Neurospora]

Supplemental material is available at http://www.genesedev.org.

Received April 28, 2005; revised version accepted August 25, 2005.

Daily and seasonal changes in light and temperature define major boundaries for the evolution of life on earth (Hastings 2000; Dunlap and Loros 2004). On a temporal level, the rhythmic environment has promoted the evolution of circadian clocks, which are cellular timekeepers that help organisms to synchronize and phase their physiology and behavior to appropriate times of the day or year. In recent years a concerted effort to unravel the molecular basis of the circadian clockwork and its connecting pathways has led to major insights into how rhythmicity is generated, how the clockwork is reset, and how the clock transduces information to generate overt rhythmicity (Dunlap 1999; Young and Kay 2001; Golden 2003). Initially, many of our insights into the molecular generation of self-sustained rhythmicity were gained by studying circadian clocks in their unperturbed, free-running state; i.e., when running in constant conditions. In all organisms studied under these conditions, a set of clock genes and their products interact in a net-

${ }^{3}$ Corresponding author.

E-MAIL christian.heintzen@manchester.ac.uk; FAX 0161-275-5656. Article and publication are at http://www.genesdev.org/cgi/doi/10.1101/ gad.349305. work of transcription/translation-based positive and negative feedback loops (TTFLs) to generate rhythmicity (Dunlap 1999; Cermakian and Sassone-Corsi 2000; Young and Kay 2001). Subsequently, a number of pioneering studies investigated the perturbing effects of light on the free-running molecular clockwork, revealing how clocks can be reset by discrete light pulses through rapid induction or degradation of clock components (Crosthwaite et al. 1995; Shigeyoshi et al. 1997; Young and Kay 2001; Ashmore and Sehgal 2003).

With a detailed knowledge of the molecular components of the free-running oscillator at hand, we can now begin to investigate how circadian clocks function under the more realistic conditions of a light-dark (LD) cycle (Roenneberg et al. 2003). For example, how can circadian clocks that are normally very sensitive to light and are quickly driven into an arrhythmic state in continuous light (LL) persist in LD cycles and control the phase (i.e., time of day) of a clock-controlled event? It was proposed that a solution to this paradox might lie in the desensitization of photo-transduction pathways during chronic light treatment (as opposed to discrete light pulses) (Johnson et al. 2004). In this model, a discrete light pulse given in constant darkness (DD) could reset the oscilla- 
tor, whereas a chronic light treatment would not, as the sensitivity of light-input pathways to the oscillator is muted.

We have previously identified a protein called VIVID (VVD) that fulfils the criteria to act as a desensitizer of light-signaling pathways. VVD shows high sequence similarity to other PAS/LOV chromophore-binding domains, with highest homology to the PAS/LOV domain of WC-1 (Heintzen et al. 2001; Christie et al. 2002; Imaizumi et al. 2003). Among other phenotypes, vvd mutants display a marked 4-h delay in clock-controlled conidiation after a light-to-dark step, suggesting a role in influencing clock phase under entrainment conditions. VVD itself has multiple functions in light signaling. Within the very early light response, VVD likely represses lightinput pathways by interfering directly or indirectly with the formation or action of the WHITE-COLLAR Complex (WCC) (Heintzen et al. 2001). More recent data have revealed that, reminiscent of WC-1, VVD also binds a flavin-derived chromophore within its PAS/LOV domain and is a WC-1-dependent photoreceptor that senses changes in light intensities (Cheng et al. 2003; Schwerdtfeger and Linden 2003).

Here we have probed VVD's role within the circadian system of Neurospora and show that VVD has a dual role in clock resetting. First, VVD prevents clock resetting at dawn, by suppressing light responses in the early hours of the day; second, VVD promotes clock resetting at dusk by enhancing frq mRNA turnover. In doing so, VVD plays a dominant role in facilitating entrainment of the circadian clock to real life conditions and in establishing the phase of the circadian clock; i.e., the time of day when developmental switches are thrown in Neurospora.

\section{Results}

VVD sustains a circadian clock that runs in the light

Circadian clocks are light sensitive, and overt rhythmicity in organisms such as Drosophila and Neurospora is lost in LL, presumably due to constant resetting of their circadian clocks. These observations pose a paradox as in real life these clocks would only function during one half of the photoperiod, effectively turning them into hourglasses rather than real clocks. In other words, if the Neurospora circadian clock is to operate in real life, its light sensitivity must be muted during the day. Our previous work had established that VVD strongly mutes clock resetting to short pulses of light. We wondered if VVD could play a similar role in LL, serving to establish a functional clock that operates in full photoperiods by preventing chronic light resetting. To test this hypothesis, we first needed to investigate the validity of its premise; i.e., the persistence of clock function during chronic light treatment. Toward this end we synchronized race tube cultures of Neurospora wild-type strains to different LD cycles before releasing cultures into LL. We reasoned that previous attempts to identify a running clock in LL might have failed because cultures were immediately exposed to LL, thus preventing manifestation of a functional feedback loop, whereas our protocol establishes an entrained clock before cultures are released into LL. Using this protocol, conidiation in wild-type Neurospora shows clear rhythmicity for $1-2 \mathrm{~d}$ in LL before becoming arrhythmic (Fig. 1A; Supplementary Fig. $\mathrm{S} 1$, middle panels, blue traces). To test whether this initial rhythmicity in LL requires a functional $v v d$ gene, we repeated the above experiments with a $v v d$ knockout strain and found that conidial banding in LL, as seen in the wild type, is lost in $v v d$ mutants in all but one (LD 4:20) (see Supplementary Fig. S1) entrainment program (Fig. 1A, middle panels, gray traces). Evidently the presence of VVD is crucial for the sustained conidial rhythmicity in the light following most entrainment conditions.

It is clear that rhythmic conidiation in the wild type is present in the first day of LL; however, the amplitude of
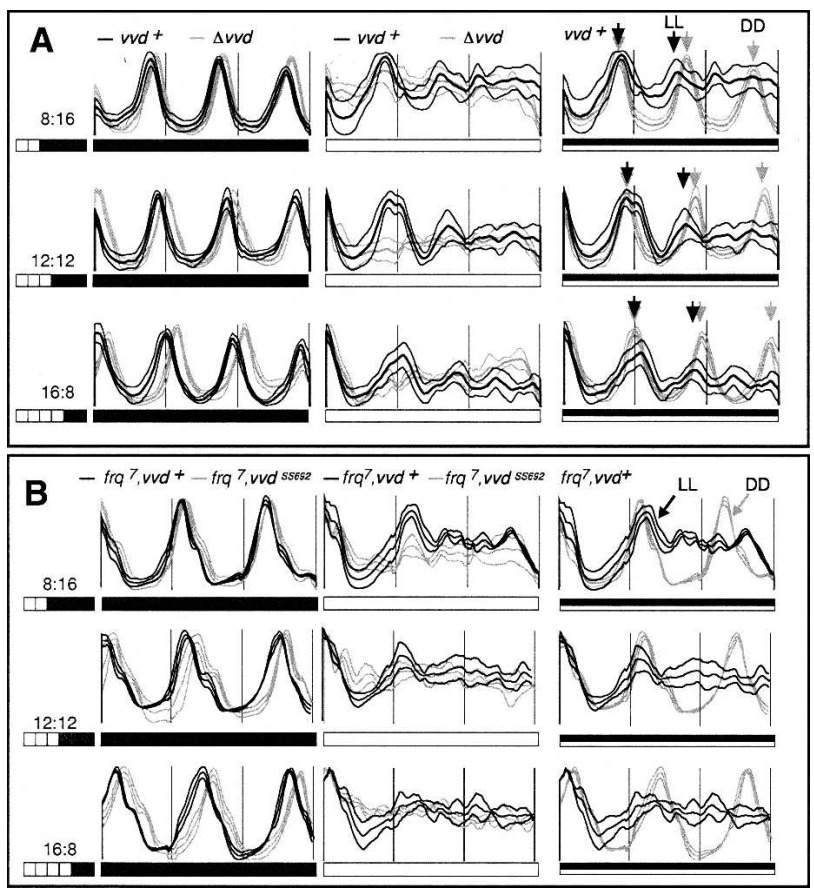

Figure 1. $\quad v v d$ permits a damped circadian clock to control conidiation in LL by preventing clock resetting at dawn. Averaged densitometric traces of 12-18 race tubes entrained for at least 2 d to photoperiods ranging from LD 8:16 to LD 16:8 before being released into DD (all left panels) or LL (all middle panels). The full set of investigated photoperiods are shown in Supplementary Figure S1. The right panels show DD and LL plots superimposed with alignment at dusk (LL plots), and subjective dusk (DD plots), respectively. (A) Race tubes were inoculated with either wild-type (black traces) or $v v d^{\mathrm{KO}}$ strains (gray traces). In the right panels, arrows indicate peaks of conidiation in wild type in DD (gray arrows) and LL (black arrows), respectively, to highlight coincident and diverging conidial rhythmicity. (B) As in $A$, but race tubes were inoculated with either $f_{r} q^{7}$ (black traces) or $\mathrm{frq}^{7}, \mathrm{vvd}^{\mathrm{SS} 692}$ double mutants (gray traces). (Thick lines) Mean; (thin lines) \pm 1 SD; (white bars) light; (black bars) darkness. 
the rhythm rapidly decreases and conidiation eventually becomes arrhythmic. Significantly, when LL cultures are compared to their corresponding DD controls, the first conidial band seen in LL precisely superimposes on the band produced in DD (Fig. 1A; Supplementary Fig. S1, right panels), suggesting that the clock, quite unexpectedly, is running into the light period without being reset at the dark-light (dawn) transition. This observation contradicts simple models for clock resetting in this organism (Crosthwaite et al. 1995). We repeated the entrainment experiment with the long period $(29 \mathrm{~h}) \mathrm{frq}^{7}$ mutant, reasoning that banding in LL should follow a long period rhythm if under control of the frq locus; i.e., the circadian feedback loop. As seen in Figure 1B (middle panel), rhythmicity in LL is indeed influenced by the frq locus. Again, the first conidial bands produced in LL and DD superimpose (Fig. 1B, right panels), indicating that the clock is not reset at dawn and instead is protected from the resetting effects of light. The period and phase differences between wild-type, $f r q^{7}$, vvd knockout, and $\mathrm{frq}^{7}$; vvd double-mutant strains are obvious when superimposed (Supplementary Fig. S2). As predicted, period length is under control of the frq locus, and similar to the results obtained with single vvd knockout mutants, the conidiation rhythm in LL in $\mathrm{frq}^{7}$; Vvd double mutants rapidly disappears (Supplementary Fig. S2, middle and right panels).

The temporary existence of overt rhythmicity in LL and the frq-dependent period effects are consistent with the interpretation that the frq oscillator continues to operate during the first day of LL without being reset at dawn. Moreover, the absence of LL rhythmicity in $v v d$ mutant strains suggests that $v v d$ is required for the manifestation of this oscillator, presumably by preventing clock from resetting at dawn and throughout the day. To obtain an independent assessment of the existence of a circadian clock in LL and its dependency on the $v v d$ gene, we entrained wild-type and vvd knockout strains to 12:12 LD cycles before releasing race tube-grown cultures to increasing day lengths (Fig. 2). Such release experiments have been used to study the contributions of dusk or dawn to entrainment and to reveal whether a circadian oscillator persists during daylight (Pittendrigh 1981; Gooch 1985; McWatters et al. 2000). Indeed, release experiments by Gooch (1985) suggested the existence of temporary clock function in days of up to $12 \mathrm{~h}$ long in Neurospora. Therefore, we predicted that if a clock is running in LL and $v v d$ is required for its existence, we should see systematic phase differences of the DD rhythm in release experiments only in wild type but not in $v v d$ mutant strains.

After lights off (4-48 h of LL), we analyzed the phase of the clock by monitoring conidial banding in the subsequent dark period. If the frq oscillator is intact in LL, we predicted systematic variations of clock phase (time of conidiation) in DD based on the previous length of photoperiod (Fig. 2A, top panel), whereas in the absence of a functional oscillator in LL (as predicted for the $v v d$ knockout strains), we expected a more or less fixed phase of circadian conidiation set at the dusk transition (Fig.
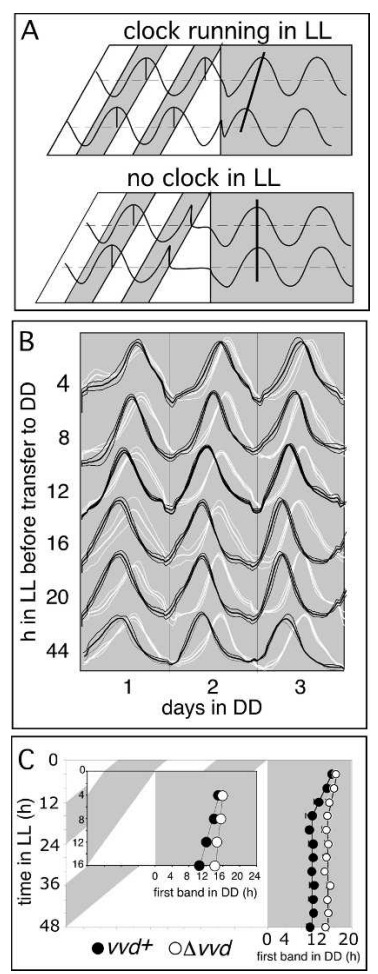

Figure 2. $\quad v v d$ controls photoperiod-dependent changes in clock phase in DD. (A) Theoretical outcome of a release experiment revealing the existence (top panel) or absence (bottom panel) of clock function in LL. After entrainment to LD 12:12 cycles, Neurospora is released into LL of increasing lengths before its free-running rhythmicity is evaluated in DD. (Top panel) In the presence of a clock running in LL, the phase of the subsequent DD rhythm will depend on the phase of the LL clock at the dusk transition. Clock phase as measured in DD will systematically change depending on the previous day length. (Bottom panel) In the absence of an LL clock, the phase of the freerunning rhythm in DD is independent of day length and is always set to the same phase at the dusk transition. (B) Averaged densitometric traces of wild-type ( $v v d^{+}$, black traces) and $v v d^{\mathrm{KO}}$ $(\Delta v v d$, white traces) strains cultured in a release experiment as depicted in $A$. (Thick lines) Mean; (thin lines) \pm 1 SD. (C) Phase of first conidial band in DD in $v v d^{\mathrm{KO}}(\Delta v v d$, open circles) and wild-type $\left(v v d^{+}\right.$, filled circles) strains, as obtained in the race tube assays described in $B$. (Inset) Enlarged data set for race tubes released in up to $16 \mathrm{~h} \mathrm{LL}$.

2A, bottom panel). As shown in Figure 2B and C, in wild type there is a marked change in clock phase as photoperiods are increased up to $16 \mathrm{~h}$, whereas in longer photoperiods clock phase is locked to dusk. In contrast, changes in clock phase are small in a $v v d$ knockout, with only $\sim 1$-h differences observed across the whole spectrum of day lengths. Thus, consistent with our previous experiments and our model depicted in Figure 2A, our data suggest that the circadian clock escapes resetting at dawn and exists throughout photoperiods of up to 12-16 $\mathrm{h}$ of LL in wild-type Neurospora. In longer photoperiods, however, wild-type strains track dusk such that clock phase always occurs at a fixed interval after lights off (Fig. 2C). This observation is consistent with previous 
interpretations that the wild-type circadian clock stops after $12-16 \mathrm{~h}$ in LL and resumes its oscillation in DD from a fixed timepoint (Gooch et al. 1994). In stark contrast, the $v v d$ knockout strain largely tracks dusk in all tested photoperiods (Fig. 2B,C) a situation best described by our model depicted in the bottom panel of Figure 2A, in which there is no or only very low amplitude rhythmicity in LL. This interpretation is further supported by the arrhythmia of $v v d$ knockout strains in LL as described earlier (Fig. 1A,B; Supplementary Fig. S1).

The absence of a robust clock in LL in $v v d$ knockout strains in all but the shortest photoperiods (Fig. 1A; Supplementary Fig. S1, middle panels) is most easily explained by the previously reported strong phase response of $v v d$ mutants to light and suggests that the clock in vvd knockout strains is reset to a state where circadian organization is breaking down. If VVD prevents clock resetting in extended light, we predicted that the phase response to light in the wild type should be strongly muted or absent during the first day of DD. To investigate this, we exposed free-running race tube cultures to brief saturating light pulses (5 min, 1750 lux) every $2 \mathrm{~h}$ during the first and second days of darkness. The effect of these light pulses was then assessed by comparing the phase of clock-controlled conidiation of light-pulsed with those of unpulsed control cultures (Fig. 3A). Plotting these data as a phase response curve (PRC) reveals that the response to light pulses in the wild type is strongly muted within the first day of LL, whereas $\mathrm{VVd}$ knockout strains respond as strongly to light in the first day as they do in the second day of darkness. These data are consistent with muted light-resetting responses seen during the first day in DD in Neurospora, an observation made by Dharmananda (1980) 25 years ago, and are conclusive evidence that it is indeed VVD that brings this about. Although the molecular mechanism whereby VVD mutes light resetting in the first day in DD is unknown, the expression profile of $v v d$ RNA in DD suggests a possibility (Fig. 3B, top panel). In DD vvd RNA shows a circadian expression profile for a single day with peak expression around DD 12 and rapidly declining levels thereafter. To the extent that $v v d$ RNA closely resembles VVD protein expression (see Fig. 3B, middle and bottom panels; Heintzen et al. 2001; Schwerdtfeger and Linden 2003), VVD would only be expressed within the first day in DD but not afterward, a profile well suited to bring about a reduced light response in the first day in DD. In summary, by repressing light signaling, and consequently clock resetting at dawn, VVD effectively allows the clock to free run into the day. Without VVD, the strong light response at dawn resets the clock into arrhythmia, a state characteristic of long photoperiods.

\section{The LL oscillator is different from the classical FRQ/WCC oscillator}

We guessed that the newly discovered rhythmicity in LL would be most easily explained by the presence of the regular FRQ/WCC TTFL that was permitted to run in the first day in LL through the repressive action of VVD
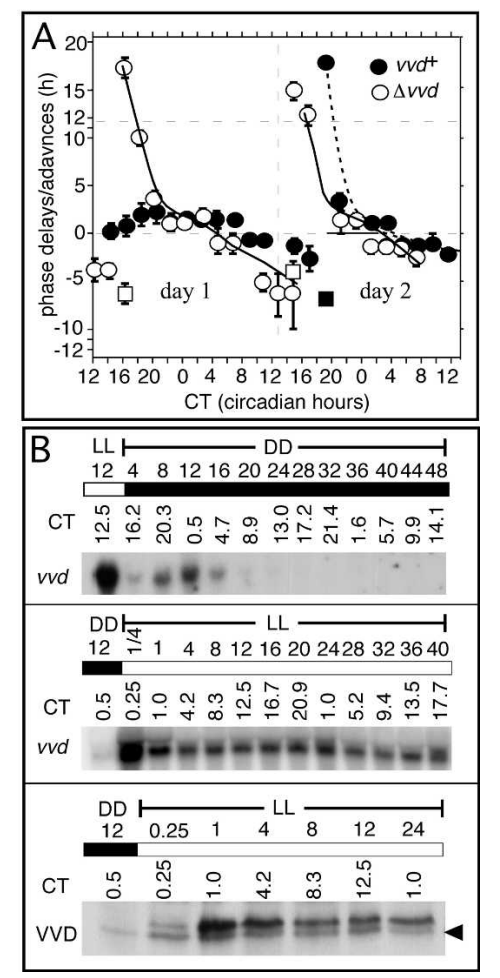

Figure 3. $v v d$ silences the resetting response to a standard light pulse during the first day in DD. (A) The light pulse PRC was obtained as described in Materials and Methods. The PRC is plotted in circadian hours, taking into account the 4-h phase difference between both strains. The center of the conidial band was defined as CT0; i.e., subjective dawn. The PRC is measured as the difference in the position of the conidial band with respect to unpulsed controls. (Abscissa) Circadian time (CT) in hours when light pulse was given; (ordinate) phase shift in hours of advance or delay; (filled circles) wild type $\left(v v d^{+}\right)$; (open circles) $v v d^{\mathrm{KO}}(\Delta v v d) ;$ (solid lines) trend lines for $\Delta v v d_{;}$(dashed lines) trend lines $v v d^{+}$PRC; (error bars) $\pm S D ; n=6-18$. (B) Northern and Western blot analysis of $v v d$ RNA and VVD protein expression in DD and LL in wild type. Cultures were grown as described in experimental conditions, and RNA or protein was extracted from samples spanning 1-2 d in DD or LL. Sampling times are given both in real hours in DD or LL or CT. The top and middle panels show $v v d$ expression profile in DD and LL, respectively. (Bottom) VVD protein levels in DD 12 and the following day in LL. Forty micrograms of total RNA and $100 \mu \mathrm{g}$ of protein were loaded per lane. Northern blots were hybridized with a radiolabeled $v v d$ probe and Western blots with an antiserum directed against the VVD ORF. The black arrowhead indicates an unspecific hybridization signal, which is also present in $v v d^{\mathrm{KO}}$ strains (data not shown). (White bars) Light; (black bars) darkness.

on the WCC. To test whether it is indeed the TTFL that operates in daylight, we first asked whether components of the feedback loop show rhythmic changes in steadystate levels in LL. We first analyzed the LL kinetics of $f_{r} q$ and other clock-controlled transcripts, including $v v d$ in wild-type and $v v d$ knockout strains (Fig. 4A,B). Strains were entrained to 12:12 LD cycles before their release into LL and subsequent tissue harvest. We found no evi- 

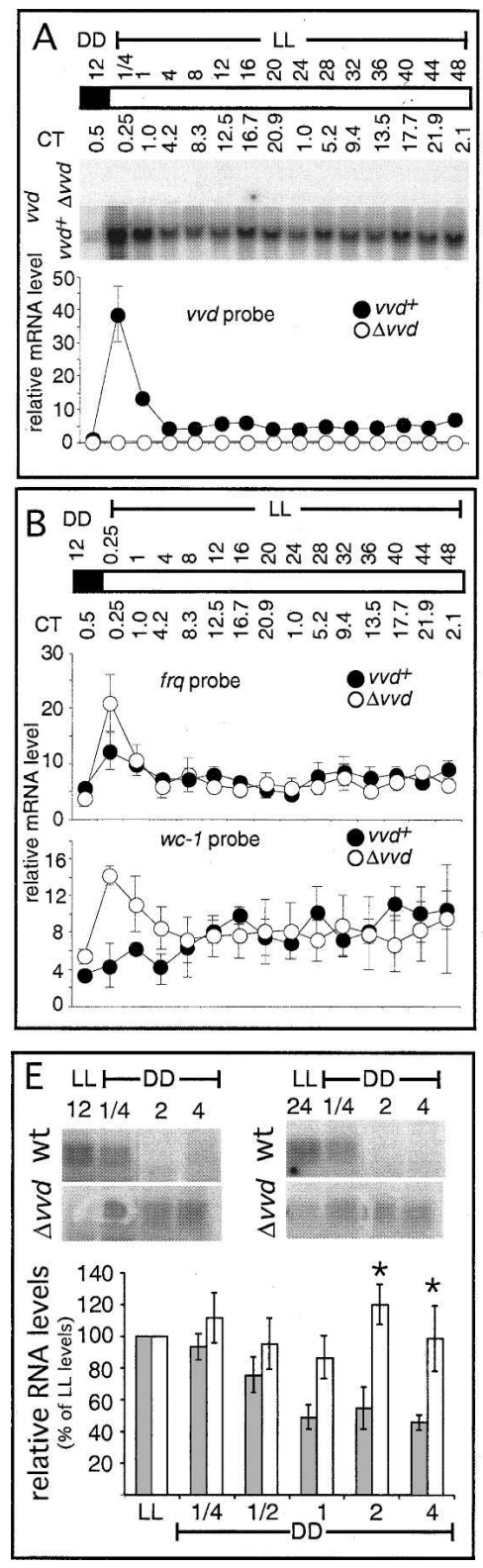

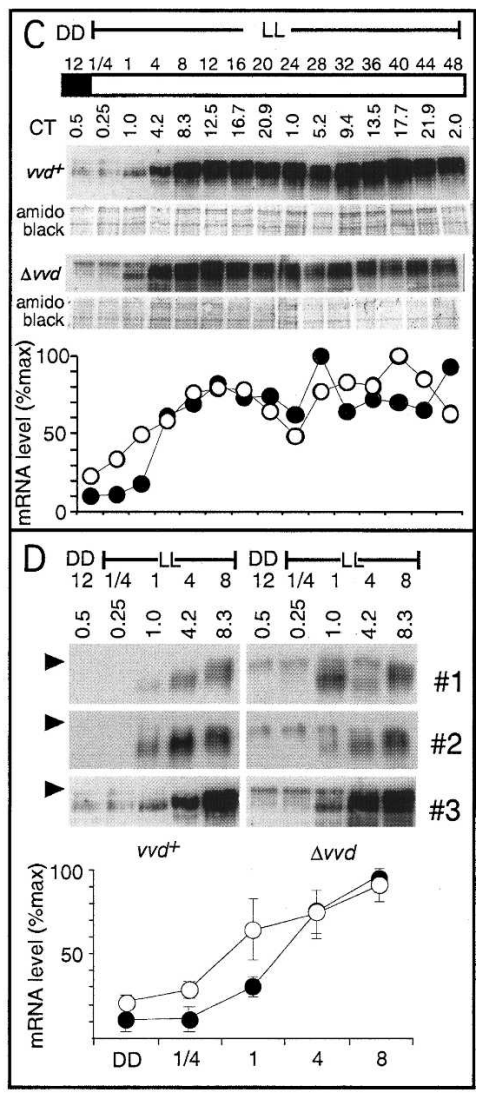

Figure 4. The classical FRQ/WCC oscillator stops early in LL, and $v v d$ helps to set clock phase at dusk. $(A, B)$ Northern blot and densitometric analysis of $\mathrm{vVd}$, $w c-1$, and $f r q$ transcripts in wild-type $\left(v v d^{+}\right)$or mutant $(\Delta v v d)$ strains $(n=3)$. Cultures were subjected to various lengths of light (see Materials and Methods) before harvest and RNA or protein extraction. Controls were kept in DD. Forty micrograms of total RNA were loaded per lane. Transcript levels are given in arbitrary units. (Filled circles) Wild type; (open circles) $v v d^{\mathrm{KO}}$ strains; (white bars) LL; (shaded bars) DD; (error bars) \pm 1 SE. (C, top panels) Representative Western blots (top) and their quantitation (bottom) showing FRQ accumulation in LL in wild type $\left(v v d^{+}\right)$and $v v d^{\mathrm{KO}}(\Delta v v d) .(D)$ Western blots (top three panels) and their quantitation (bottom) showing FRQ protein levels in the first $8 \mathrm{~h}$ in LL (three independent experiments) to emphasize differences in FRQ protein accumulation between mutant and wild type during the early light response. The arrowhead highlights the different FRQ form accumulating in $v v d^{\mathrm{KO}}(\Delta v v d)$. One-hundred micrograms of protein were loaded in each lane. $(E)$ Northern analysis of frq expression levels in DD after exposure to different photoperiods (LL 12 and LL 24). Cultures were grown as described in Materials and Methods, and RNA was extracted from control samples before lights off (LL) and over a period of $4 \mathrm{~h}$ into subsequent DD. Northern blots were hybridized with a radiolabeled vvd probe. The densitometric analysis from three replicate experiments of a more detailed time-course is shown below. RNA levels are given relative to LL values, which were arbitrarily set to $100 \%$. (Dark bars) Wild type $\left(v v d^{+}\right)$; (white bars) $v v d^{\mathrm{KO}}(\Delta v v d)$; (error bars) $\pm 1 \mathrm{SE}$; (asterisks) differences that are statistically significant at the 0.05 confidence level. dence for molecular rhythmicity at the transcript level for frq or any of the other investigated genes, suggesting that the frq-influenced rhythmicity during daytime is most likely maintained at the post-transcriptional level. As previously reported, all transcripts are light induced, with maximal induction between $15 \mathrm{~min}$ and $1 \mathrm{~h}$ after lights on, albeit $w c-1$ induction in wild type was weak in our experiments. In the absence of transcript oscillations, we asked whether FRQ protein levels might dictate the LL rhythm. Figure 4C (top panels) shows a gradual increase of FRQ protein levels in LL in both wildtype and $v v d$ mutant, peaking only after $12-16 \mathrm{~h}$ in LL, consistent with previous observations in wild type (Collett et al. 2001; Tan et al. 2004a). These data suggest that the initial phase of rhythmic conidiation in LL most likely arises from changing FRQ protein levels, but other as yet undiscovered processes may contribute to the ex- tended overt rhythmicity we see in LL. We see little quantitative difference in FRQ protein accumulation between wild-type and $v v d$ knockout strains, but there are qualitative changes as larger forms of FRQ appear to be more prevalent during the first 4-8 h of LL in the $v v d$ knockout strain (Fig. 4D). We see no obvious rhythmicity of WC-1 protein in LL (data not shown), suggesting that, although quantitative as well as qualitative changes in the TTFL might help to influence rhythmic conidiation in LL, other components or mechanisms are likely to bring about extended rhythmicity in LL.

\section{VVD influences the phase of the clock in $D D$ by promoting clock resetting at dusk}

Previously we reported that Neurospora strains, mutated in the $v v d$ gene, enter free-running conditions of DD 
with a phase delay of $\sim 4 \mathrm{~h}$ (Heintzen et al. 2001). Our release experiments (Fig. 2) offer a straight-forward explanation for this observation in which the phase differences between both strains arise simply due to the presence or absence a functional oscillator during daylight. Thus, the maximum 4-h phase differences observed earlier are only established in photoperiods $>20 \mathrm{~h}$ LL. As expected, such photoperiod-dependent phase differences are also seen between $f_{r q^{7}}$ and $f r q^{7}, v v d$ double mutants (Fig. 1B). However, when comparing levels of clock transcripts and proteins toward the end of light periods $>20$ $\mathrm{h}$, we do not see any significant differences between wild-type and $v v d$ knockout strains (Fig. 4A-C) that could explain the phase differences seen in overt rhythmicity. Thus, based on the molecular data, wild-type and mutant clocks should enter DD with roughly the same phase. In other words, the large phase differences in overt rhythmicity between wild type and mutant cannot be easily explained by differences in clock gene expression at the end of long light periods. We therefore wondered if changes in gene expression immediately following the dusk transition (rather than preceding it) could account for the delay in overt rhythmicity. To test this hypothesis, we investigated $f_{r} q$ RNA kinetics within the first 4 $\mathrm{h}$ in DD after Neurospora liquid cultures had been exposed to photoperiods of 12 and $24 \mathrm{~h}$, respectively (Fig. $4 \mathrm{E})$. It is clear from Figure 4E that, in all conditions tested, frq RNA levels in $v v d$ knockout strains are elevated for much longer compared with wild type, despite starting off with more or less the same levels at the dusk transition. Thus, the presence of VVD promotes a more rapid turnover of $f r q$ RNA in wild-type strains, quickly setting the clock to CT12 (subjective dusk), whereas in the absence of VVD, resetting to frq trough levels is prolonged by $\sim 4 \mathrm{~h}$.

\section{Overexpression of VVD rescues clock phase defects of the vvd knockout strain}

To confirm VVD's role in regulating clock phase, we set out to constitutively express VVD in a $v V d$ knockout background. Toward this end we fused the VVD open reading frame $(\mathrm{ORF})$ to the inducible qa-2 promoter (Fig. 5A) whose activity can be induced by the carbon source quinic acid (Tyler et al. 1984; Aronson et al. 1994a). A number of transformants showed successful insertion of the construct at the his locus (Fig. 5B) and rescued the phase defect of the knockout strain when grown on quinic acid medium (data not shown). When the primary transformants were backcrossed into the parental strain (289-10; $\Delta v v d, b d$, his-3) to obtain homokaryotic progeny, siblings segregated according to Mendelian genetics with only those progeny that carried the transgene able to rescue the phase defect; i.e., advancing phase $\left(\chi^{2}=0.143<3.84\right)$. The phase advances seen are statistically significant (one-way ANOVA, $P=0.0001$ ). As expected, the free-running period (FRP) of both parent (289-10, FRP $=25.1 \mathrm{~h})$ and $q a-2: \mathrm{VVD}$ transformants $(\mathrm{FRP}=25.0 \mathrm{~h})$ are long, due to the presence of histidine in the growth medium, but are not significantly different

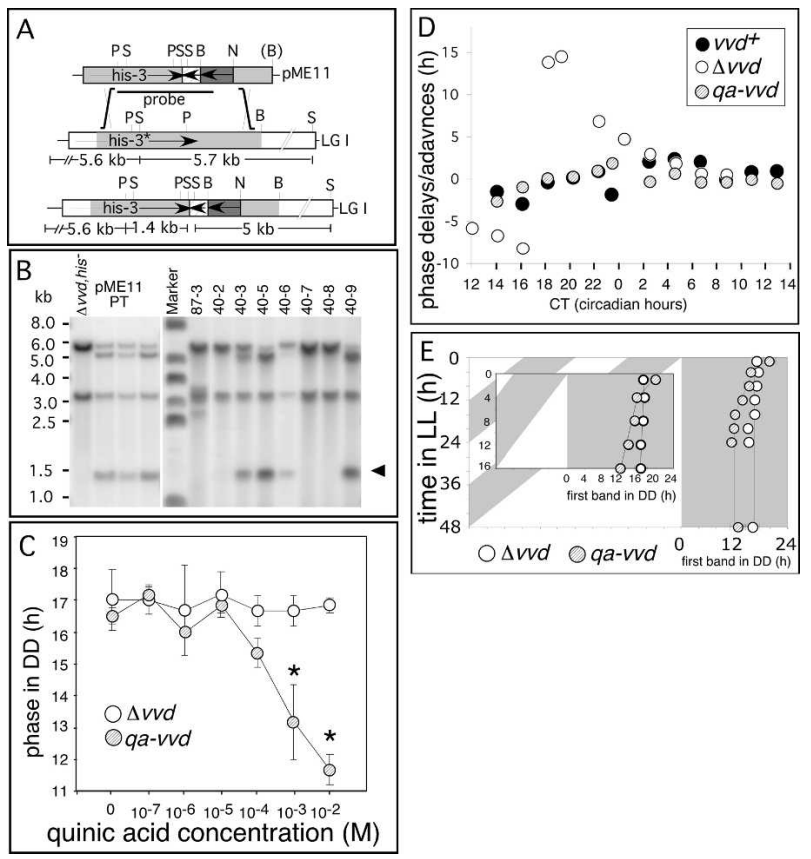

Figure 5. Constitutive VVD expression rescues clock-phase and light-resetting defects in vvd knockout strains. (A, top) Generation of strains that express qa-2-inducible VVD. (Top) Plasmid pME11 contains a translational fusion of the $q a-2$ promoter (shaded dark gray) to the $v v d$ ORF (hatched area), inserted into $5 \mathrm{~kb}$ of genomic his-3 region, which is available for homologous recombination. (Middle) Genomic organization of his-3 locus of parental strain 289-10 ( $\Delta v v d$, bd, his). (Bottom) Genomic organization of $q a-v v d$ knock-in strain created by homologous recombination at the his locus. Predicted SalI fragments are shown. (LG I) Linkage group I; (B) BamHI; (N) NotI; (P) PstI; (S) SalI; (arrows) direction of transcription. (B) Southern blot analysis of SalI-digested genomic DNA of primary transformants (pME11PT) and progeny from backcrosses to the $\Delta v v d$, his ${ }^{-}$parent (40-2 to 40-9). The arrow indicates the position of the expected genomic fragment containing the inserted $v v d$ gene. $(C)$ Phase of the first band of conidiation in DD of parental $v v d$ knockout strain $(\Delta v v d$, open circles) and a representative $q a-v v d$ knock-in strain (hatched circles) grown on increasing concentrations of the inducer quinic acid. Race tubes were grown for $2 \mathrm{~d}$ in LL before transfer to DD. Clock phase was analyzed for three independent experiments and plotted against quinic acid concentration. (Error bars) \pm 1 SD. $(D)$ Phase response of wild type $\left(v v d^{+}\right.$, filled circles) $v v d^{\mathrm{KO}}(\Delta v v d$, open circles), and a $q a-v v d$ knock-in strain (hatched circles). The light pulse PRC was obtained as described in Figure 3A. (Abscissa) Circadian time $(\mathrm{CT})$ in hours when light pulse was given; (ordinate) phase shift in hours of advance or delay. (E) Photoperioddependent phase is rescued in a qa-vvd knock-in strain. The experimental procedure is the same as described in Figure 2C. (Inset) Enlarged data set for race tubes released in up to $16 \mathrm{~h} \mathrm{LL}$.

from each other (two sample $t$-test, $t=1.05, P=0.3197$ ). After verifying that transformants carrying the $q a$ 2:VVD construct express the $v v d$ gene in a quinic aciddependent manner (data not shown), we examined the dependence of phase rescue on the concentration of quinic acid added to the medium (Fig. 5C). We found that increasing the quinic acid concentration induces increas- 
ing phase advances within the qa-2:VVD strains, whereas the phase of the parental $v v d$ knockout strain is quinic acid insensitive. Again, the changes seen are statistically significant for the two highest quinic acid concentrations used (two-way ANOVA: QA concentration, $P=0.0251$; strains, $P=0.0001)$. Quinic acid concentrations of $10^{-4} \mathrm{M}$ quinic acid and below are ineffective, however, and only after reaching a critical threshold of $10^{-4} \mathrm{M}$ is phase seen to move forward in the qa-2:VVD "knock-in" strains. Again, there is no significant effect of the various quinic acid concentrations or the analyzed strains on the observed FRP (two-way ANOVA: qa concentration, $P=0.3922$; strains, $P=0.7574)$. Moreover, as shown in Figure 5D, the increased sensitivity of $v v d$ mutants to light pulses during the first day of DD is restored in $q a-2: V V D$ strains to the characteristic wild-type light response that is highly muted in the first day of DD (Fig. $3 A)$. Finally, subjecting the $q a-2:$ VVD strain to increasing photoperiods shows that ectopic insertion of VVD can rescue the photoperiod-dependent changes in clock phase, as seen in the wild type (Fig. 5E). The inset in Figure 5 highlights the strong dependency of phase on conidiation in qa-2:VVD strains, following photoperiods of up to $20 \mathrm{~h}$ in length. As expected, following photoperiods of up to $20 \mathrm{~h}$, the slope generated by the photoperiod-dependent phase changes is significantly different from the slope seen for the $v v d$ knockout parent (two sample $t$-test: $t=-6.16, P=0.0000)$. More importantly, the slope seen for $q a-2: V V D$ strains is significantly different from 0 (slope $=-0.24, t=-10.01, P=0.0002$ ), as expected, if photoperiodic history influences clock phase. In contrast, the slope seen for $v v d$ knockout strains is not significantly different from 0 , indicating that they resume from a fixed phase at dusk regardless of the preceding photoperiod (slope $=0.079, t=-2.33$, $P=0.0676$ ). In both strains the phase of circadian conidiation becomes locked to dusk following photoperiods $>20 \mathrm{~h}$ (vvd knockout: slope $=0.003, t=0.19$, $P=0.8603$; qa-2:VVD: slope $=0.06, t=1.96, P=0.1073$ ), suggesting that their clocks have stopped or oscillate with only a very shallow amplitude. These results are important for two reasons: First, they show that all clock-specific phenotypes of the parental knockout strain can be rescued by reinserting the $v v d$ gene into a vvd knockout parent. Second, despite constitutive expression of VVD, clock phase in the qa-2:VVD strains is still photoperiod dependent. Thus, in line with the constitutive expression of VVD in extended periods of light (Fig. 3B, middle and bottom panels) the quinic aciddriven constitutive expression of VVD will rescue all its clock-related functions.

Given VVD's influence on clock resetting at both ends of the light period, one can expect profound effects in vVd mutants on the entrainment behavior of the Neurospora clock to complete $24-\mathrm{h}$ photoperiods. Figure 6A provides supporting evidence for this assumption. In an LD 12:12 cycle, rather than conidiating just once during a full entrainment cycle (as wild type does), the $v v d$ knockout strains show a clear second peak of conidiation that occurs well into the light period. This second peak

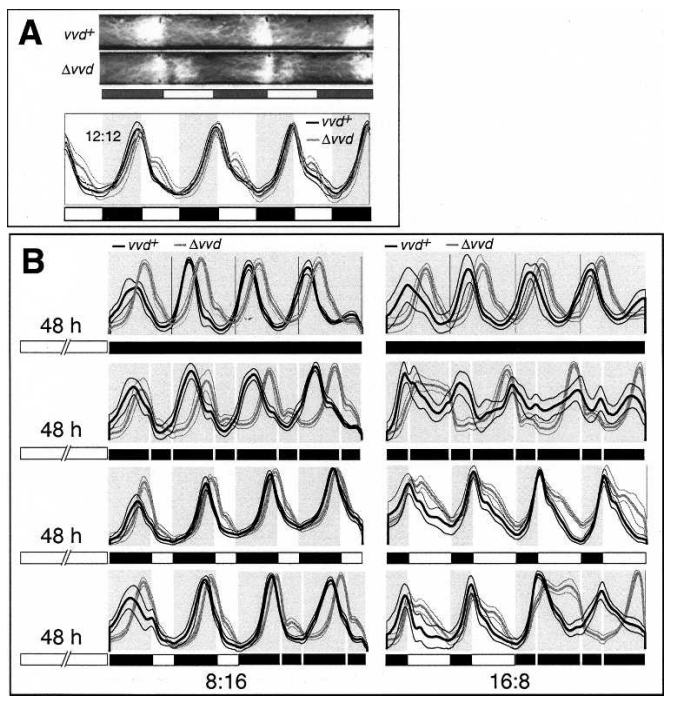

Figure 6. VVD facilitates entrainment to continuous photoperiods. (A) Averaged densitometric traces of race tubes inoculated with wild type (87-3, black traces) or $\Delta v v d$ (289-1, gray traces) grown in LD 12:12 entrainment conditions; $n=6-18$. Plots are aligned at the dark-light boundaries. (Thick line) Mean; (thin lines) $\pm 1 \mathrm{SD}$; (white bars) light; (black bars) darkness. Note the second peak of conidiation in the $\Delta v v d$ strain. (B) Averaged densitometric traces of race tubes inoculated with wild-type (87-3, black traces) or $\Delta v v d$ strains (289-1, gray traces) grown in skeleton or complete photoperiods. (Top panels) DD controls. (Second panels) 8:16 (left) and 16:8 (right) skeleton photoperiods. (Third panels) 8:16 (left) and 16:8 (right) complete photoperiods. Note the different phases of conidiation under skeleton and complete photoperiods in wild type in 8:16 photoperiods. $\Delta v v d$ strains show similar phases of entrainment in both conditions. Also note that $\Delta v v d$ strains always interpret the long dark interval between light pulses as night. (Bottom) LD 8:16 (left) and LD 16:8 (right) complete photoperiods, followed by corresponding skeleton photoperiods. Note the phase reversal of $\Delta v v d$ strains that is not seen in wild type. For a more detailed discussion of this figure, see Results and Discussion.

can be interpreted in two ways. First, it could arise as a consequence of the failure to prevent clock resetting at dawn, leading to a large phase advance of the clock, as predicted from the light PRC (Fig. 3A). Second, the double-peak of conidiation in a $v V d$ knockout strain could arise from a transient suppression of conidiation at the onset of the light period, triggered by some acute effect of light that temporarily bypasses clock control of conidiation. In this scenario, conidial banding in the mutant could be interpreted as delayed conidiation, a phenotype predicted from the absence of VVD's function in influencing clock resetting at the dusk transition (Fig. 2B).

The apparent absence of clock resetting at dawn and the presence of a clock in complete LD cycles suggest that Neurospora may entrain to full photoperiods by way of continuous entrainment. Models of continuous entrainment propose that light acts continuously (chronically) on the oscillator to bring about entrainment and to establish the phase angle (i.e., the characteristic time of 
day when clock-controlled events are turned on or off) during complete LD cycles. In contrast, discrete entrainment models propose that the dawn and dusk transitions are sufficient to explain the phase angle observed in full photoperiods (for a review on continuous and discrete models of entrainment, see Johnson et al. 2004). Therefore, if "skeleton photoperiods" consisting of brief light pulses presented at dawn and dusk can mimic complete photoperiods, one would expect little or no difference in the phase of conidiation, compared with complete photoperiods. However, if the continuous action of light is required to establish the characteristic phase angle, peaks of conidiation in complete photoperiods may be significantly different from those observed in skeleton photoperiods. We tested both models by entraining race tube-grown cultures of wild-type and $\mathrm{vvd}$ knockout strains to either 8:16 and 16:8 LD complete photoperiods, their corresponding skeleton photoperiods, and a combination of both (Fig. 6B). It is clear that the phase of conidiation of wild-type strains in complete and skeleton photoperiods is quite different. For example in 8:16 skeleton photoperiods, peaks of conidiation occur during the first half of the subjective night, whereas in complete photoperiods, the peak of conidiation occurs toward the end of the night, $\sim 8 \mathrm{~h}$ later (cf. second and third panels in Fig. 6B for 8:16 entrainment). In contrast, the $v v d$ knockout strains show little if any differences in phase; whether entrained by complete or skeleton photoperiods, vvd knockout strains conidiate late in the (subjective) night. When comparing 16:8 photoperiods, phases of conidiation in both wild-type and $v v d$ knockout strains differ when comparing the skeleton with the corresponding complete photoperiods. We anticipated this difference in phase, because skeleton photoperiods that are $>13-14$ h generally (given the right PRC) produce a phase angle jump; i.e., the organism now interprets the shorter dark interval as "subjective day" and adopts a phase similar to that seen for short skeleton photoperiods. Indeed, this phase angle jump is obvious in the vvd knockout strains that, once transferred from complete to skeleton photoperiods, very rapidly adjust conidiation to a new phase that is similar to that seen in short skeleton photoperiods (Fig. 6B, cf. bottom right panel and second panels of both skeleton photoperiods). However, wildtype strains do not show this rapid adjustment in phase, nor do they seem to entrain well under long skeleton photoperiods (Fig. 6B, second right panel). This observation is in line with the weak phase response seen in wild type (Fig. 3A). Although the length of our race tubes limit our analysis, it appears that in 16:8 skeleton photoperiods, the wild type will eventually entrain to a phase similar to that seen in short skeleton photoperiods; i.e., peaks of conidiation may occur $\sim 6-7 \mathrm{~h}$ into the long dark interval (Fig. 6B, cf. last peak of conidiation in 16:8 and peaks of conidiation in 8:16 skeleton photoperiods). However, the occurrence of additional peaks of conidiation (possibly due to acute effects of light on conidiation) makes interpretation of these traces difficult. In 8:16 skeleton photoperiods, the wild type appears to entrain better and establish a stable phase within the duration of our experiment (Fig. 6B, second left panel). Taken together, it is clear that VVD is required to establish the characteristic phase of conidiation in complete LD cycles. Generally light pulses cannot mimic the phase of conidiation seen in complete photoperiods in wild-type Neurospora, suggesting that the continuous influence of light in complete photoperiods plays an important role in determining the phase angle of entrainment. In addition, and given the complexity of control of conidiation in full photoperiods, it likely that other events, such as acute responses to light, shape the conidiation rhythm in the real world. This aside, it is clear that VVD helps to maintain the time (i.e., phase) of day when developmental switches are thrown in Neurospora in continuous photoperiods.

\section{Discussion}

We have shown here that VVD's repressive action on the WCC has two previously unnoticed, but important, consequences on the Neurospora circadian clockwork that provide insights into the molecular mechanism of entrainment to complete photoperiods. First, by muting or preventing light resetting at dawn, VVD permits the circadian clock to operate during the daytime. Second, we find that VVD, in contrast to its function at dawn, promotes resetting at dusk by influencing the rate of $f_{r q}$ RNA turnover. Thus, two surprisingly opposing effects of VVD-the first to prevent, the second to promote resetting of the circadian clock-ensure integrity and correct phase of the circadian clockwork within the complete photoperiods of real life. The release experiments (Fig. 2) suggest that the wild-type clock either stops operating within the first $12-16 \mathrm{~h}$ of LL or assumes lowamplitude oscillations that are difficult to identify in our experimental conditions. This conclusion is in line with our observation that in LL, FRQ protein levels increase for $12-16$ h but show no further obvious quantitative or qualitative changes afterward; however, it contrasts with our observation of extended rhythmicity on race tubes kept in LL. A number of scenarios may explain these observations: First, it is possible that small amplitude rhythms of a FRQ-dependent oscillator that currently lie below our detection limit are sufficient to drive overt rhythmicity for a few cycles. For example, it is conceivable that subpopulations of FRQ or WC-1 that we might miss using standard Western blot techniques may play a part in LL rhythmicity. It is also possible that rhythmic FRQ phosphorylation occurs without corresponding RNA fluctuations, and this is not without precedent in other organisms. For example, a TTFL-independent mechanism in rhythmic KaiC phosphorylation was recently uncovered in Cyanobacteria (Tomita et al. 2005) and, in fibroblast cultures and despite constitutive overexpression of mPer2, mPER2 protein levels are still robustly rhythmic (Yamamoto et al. 2005). Alternatively and second, other oscillators or slave oscillators might enhance or complete rhythmic conidiation in LL at the output level. There is mounting evidence for the existence of slave oscillators in Neurospora (Loros and Feld- 
man 1986; Lakin-Thomas and Brody 2000; Correa et al. 2003; Granshaw et al. 2003; Pregueiro et al. 2005). Whereas these slave oscillators may normally be phased and coordinated by a master oscillator, they may become more important under conditions where circadian organization would otherwise break down, such as in LL. For example, it is possible that in LL the master clock is able to operate in the first half of a full cycle but a slave oscillator is subsequently required to complete overt rhythmicity. In this respect the TTFL would be dominant over whatever other mechanism is driving rhythmicity.

Regardless of the molecular mechanism, it is clear that a functional VVD protein is required to allow overt rhythmicity in LL to become manifest. It is possible that the absence of conidial rhythmicity in $v v d$ mutants is due to a clock-independent effect of $v v d$ on conidiation. For example, a role of $v v d$ in light-controlled conidiation has been suggested before (Shrode et al. 2001). Thus, $v v d$ 's effect on conidiation may prevent us seeing a functional clock due to masking of conidial rhythmicity. If, indeed, masking occurs, one would expect vvd mutants to maintain strong photoperiod-dependent fluctuations in the phase of conidiation in DD, simply because the underlying clock mechanism, although phenotypically masked, should be able to influence rhythmicity in DD (comparable to the situation in wild type). However, this is clearly not the case, as conidial rhythmicity in $v v d$ mutants starts more or less from the same phase regardless of the previous light history.

Our release and skeleton photoperiod experiments support a model of continuous entrainment in which the continuous action of light is required to establish the correct phase of entrainment in complete photoperiods. The release experiments show that the entrained Neurospora clock functions across the dark-light boundaries in DD and LL, and the results from skeleton photoperiods suggest that this light clock is influenced by the chronic action of light (Fig. 6). So far, however, we have only tested short light pulses (15-min) in our experiments with skeleton photoperiods, and it is possible that only part of the light period is required to establish the characteristic phase angle of entrainment in full photoperiods. This aside, it is clear that the phase response to brief light pulses fails to predict the phase of the entrained Neurospora clock accurately and that a more continued action of light is required for correct entrainment.

Models of entrainment to continuous photoperiods (versus discrete skeleton photoperiods) have been proposed in the classical circadian literature (Petersen 1980; Pittendrigh 1981). For example, it was proposed that in entrainment by a LD cycle, the circadian clock continuously moves between two alternating states, one that is established in the dark and another that is established in the light. In a sense, two oscillators-one operating in the dark, the other in the light-combine to establish the entrained oscillator. Such models fit our observation and those of others that in Neurospora in LL, a circadian oscillator produces a low amplitude rhythm that centers around CT12, a phase from which the DD oscillator resumes its feedback loop at dusk (Petersen 1980; Gooch 1985; Johnson et al. 2004). A simplified depiction of how the Neurospora clock might operate according to this model is shown in Figure 7. A more topological depiction of this model, in which an oscillator is shown as a socalled dual limit cycle can be found in the Supplementary Figure S3. Although the dual limit cycle model of entrainment is widely acknowledged as a general framework for understanding entrainment, both by light pulses and by continuous photoperiods, it has gained relatively little attention (Johnson et al. 2004). This is in part due to the lack of supportive molecular data and the success of alternative models of entrainment that predict entrainment solely on the basis of discrete light pulses at dawn and dusk transitions (Pittendrigh 1981; Crosthwaite et al. 1995). However, given that most organisms are exposed to continuous photoperiods, discrete entrainment models remain special cases of entrainment that can only provide an incomplete picture of how organisms entrain to real life conditions (Johnson et al. 2004). It seems likely that both discrete and LL responses of the oscillator establish the characteristic phase of entrainment in natural conditions. For example, components that control rapid $f_{r q}$ transcription (such as the WCC) may mediate discrete entrainment as seen in skeleton photoperiods, whereas more gradual light responses

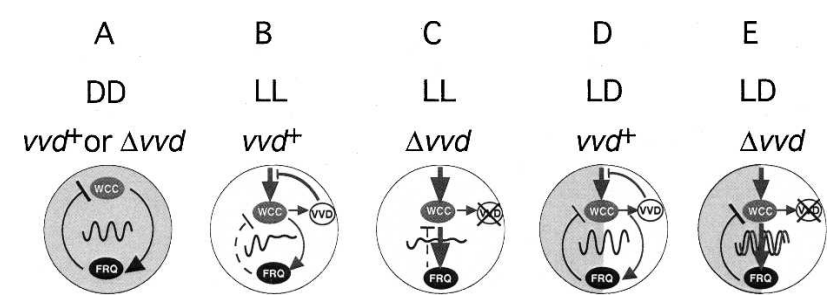

Figure 7. A molecular model for the entrained Neurospora circadian clock. $(A-E)$ For reasons of clarity, the Neurospora oscillator is depicted here as a simplified feedback loop built from the WCC and FRQ protein(s). For a limit cycle depiction of this figure, see Supplementary Figure S3. (A) In DD a robust circadian clock operates in wild-type $\left(v v d^{+}\right)$and $(\Delta v v d)$ knockout strains. $(B)$ In wild type $\left(v v d^{+}\right)$grown in LL, VVD represses lightinput pathways and prevents light resetting of the oscillator, thus allowing for the temporary formation of a functional feedback loop in LL-however, with dampened amplitude-resulting in clear circadian conidiation cycles only for the first day or two. $(C)$ In $v v d$ knockout strains $(\Delta v v d)$ growing in LL, the absence of VVD protein allows the WCC to reset the oscillator and override its negative feedback regulation by FRQ, effectively preventing the formation of a functional feedback loop. (D) In LD entrainment, a wild-type strain establishes an entrained oscillator consisting of segments of the previously described LL and DD oscillators. The phase of the oscillator at dusk, and thus the phase of circadian conidiation, is photoperiod dependent. $(E)$ In LD entrainment, a $v v d$ knockout strain $(\Delta v v d)$ is reset at dawn and the oscillator resumes at dusk at a fixed and delayed phase compared with wild type. Clock phase in $v v d$ knockout strains is largely independent of photoperiod. (Lines with arrowheads) Activating pathways; (blocked lines) inhibitory pathways; (dashed lines) partially functional pathways; (gray lines) light-signaling pathways. 
(perhaps in the activity of kinases/phosphatases that modify FRQ activity/stability) could exert a more continuous effect on the oscillator by changing its kinetics and length of cycle in response to the duration of light. In this respect it seems likely that VVD's influence on WC-1 and FRQ phosphorylation plays a role in shaping the kinetics of the feedback cycle in LL. This view of continuous entrainment assumes that the chronic action of light on the oscillator may be sufficient to bring about the characteristic entrainment to complete photoperiods. However, the classic model of continuous entrainment put forth by Aschoff and colleagues proposed that changes in light intensities during the day may continually modify the speed of the oscillator and thus confer entrainment to continuous photoperiods (for review, see Johnson et al. 2004). This model is based on Aschoff's rule that states that many day-active animals speed up their clocks with increasing light intensities, whereas night-active animals slow their clocks down under the same conditions. It is difficult to investigate this in Neurospora, first in terms of assigning the "activity type," and second because Neurospora's rhythm rapidly dampens in LL. However, comparing our release experiments at 1750 lux with those conducted at 11,000 lux seems to suggest a lengthening in FRP at higher light intensities (at least for the first cycle in LL) (Supplementary Fig. S1, cf. A and B). Given that our clock output (conidiation) occurs usually during the night, this result would thus conform with Aschoff's rule and imply that continuous entrainment in Neurospora could also follow through period adjustments via changing light intensities. It is tempting to speculate that this is indeed a mode of action in which VVD would be important, as VVD has been shown to be a photoreceptor that is required for measuring changes in light intensities in Neurospora (Schwerdtfeger and Linden 2003).

With VVD, we now have identified a molecular component that is critical for establishing this entrained oscillator and whose function rationalizes the long-standing observation of why the Neurospora clock predominately entrains to dusk (Gooch 1985; Chang and Nakashima 1997). Our release experiments (Fig. 2B,C) suggest that the Neurospora oscillator either stops in light periods $>16 \mathrm{~h}$ or oscillates around a stable phase with a very narrow amplitude that is too shallow to be detected in our experimental setup. In either case, it appears that the FRQ-WCC oscillator that drives robust rhythmicity in DD is only temporarily functional in LL, presumably because in extended photoperiods VVD can not fully repress light resetting. In consequence, chronic resetting eventually stops the clock from producing robust circadian oscillations, and following photoperiods $>12-16 \mathrm{~h}$, the DD oscillator resumes from an essentially fixed phase. In contrast, $v v d$ mutants appear arrhythmic from the start, consistent with the interpretation that the clock is immediately reset to the arrhythmic or low amplitude state. Whether rhythmicity in $v v d$ mutants is truly lost or persists with a very low amplitude is difficult to assess, but both possibilities are consistent with our results. Regardless of the underlying mechanism, vvd mutants can be considered arrhythmic in LL, as conidiation starts from more or less the same phase at dusk, regardless of the previous photoperiod. Considering that the wild-type clock runs for $\sim 12 \mathrm{~h}$ in $\mathrm{LL}$, one might ask why conidial rhythmicity in wild-type and vvd mutants is not out of phase by as much as $12 \mathrm{~h}$, and not just by $3-4 \mathrm{~h}$. Our results suggest that the phase of the LL rhythm is translated into DD clock phase mostly through frq RNA turnover kinetics. The kinetics of the frq RNA decay portion of the FRQ feedback cycle have previously been shown to only take 3-6 h (Merrow et al. 1997) and are consistent with our observation of maximum phase differences of $\sim 4 \mathrm{~h}$ at the dusk transition. Taken together, independent of the phase of the clock in LL, the phase of the DD rhythm can only maximally differ by 4-6 h.

Given VVD's influence on WC-1 phosphorylation, changes in $f r q$ turnover in $v v d$ mutants are likely to be indirect via influencing WCC activity (Talora et al. 1999; Heintzen et al. 2001; Schwerdtfeger and Linden 2001). It is conceivable, for example, that at the dusk transition, transcriptionally highly active WCC is carried over into the dark and temporarily continues to activate frq transcription. The presence of VVD would prevent such a transient activation, whereas in the absence of VVD full induction can occur, effectively causing a delaying phase shift at dusk. Indeed, we consistently observed an increase in $f_{r} q$ levels directly after the dusk transition in vvd knockout strains. In addition, previous work has shown that in LL, a hyperphosphorylated form of WC-1 predominantly accumulates in $v v d$ mutants (Heintzen et al. 2001; Schwerdtfeger and Linden 2001). In the light the hyperphosphorylated WC-1 protein is considered the transcriptionally active form of WC-1 (Talora et al. 1999). Thus, increased levels of active WC-1 at the dawn transition in $v v d$ mutants may explain the transient increase of $f r q$ transcript levels upon LD transfer.

Although it is formally possible that two principally independent clocks operate within Neurospora in DD and in LL, the frq dependency of the FRP of the circadian clock in LL (Supplementary Fig. S2) suggests that both, the DD and LL clock, utilize established clock components such as FRQ, WC-1, and WC-2 (Fig. 7). VVD's role within this system would be to mute sensitivity of the previously established (DD) clock to light resetting and thus permit its continued existence in the light (Fig. 7B). The observation that the fall in frq transcript levels is more rapid in wild-type strains compared with vvd knockout strains was implicitly predicted by Ruoff et al. (2001), who investigated the Neurospora phase response to light using a relaxation model of the Goodwin oscillator. Their modeled light PRC is in excellent agreement to published light PRCs and requires frq transcript inhibition directly after a perturbing light pulse (Ruoff et al. 2001).

The significance of photoperiod-dependent phasing of the circadian clock may lie in proper phasing of conidiation toward the end of the dark period (Figs. 6B, 7). It is generally assumed that Neurospora benefits from conidiating toward the end of the night by avoiding exposure of 
its spores to high light stress and desiccation. In this case, $v v d$ might help to maintain its proper clock phase under a wide range of photoperiods, consequently allowing Neurospora to populate a wide range of latitudes. Recent reports of photoperiodism in Neurospora (Tan et al. 2004b) may link this regulation with that observed in many plants (Yanovsky and Kay 2003; Searle and Coupland 2004).

In Arabidopsis the protein ELF3 has been implicated in repressing light responses, thus affecting its light-resetting response (McWatters et al. 2000; Covington et al. 2001; Hicks et al. 2001). In contrast to wild-type Arabidopsis, which is a facultative long-day plant that measures day length with a clock phase that is set to dawn, elf3 null mutants are arrhythmic in LL, set their clock phase to dusk, and have lost their photoperiodic flowering response (McWatters et al. 2000). Thus, ELF3 and VVD both prevent clock resetting by repressing light signaling. However, both proteins are structurally unrelated and prevent clock resetting at opposite phases of the cycle. In addition, VVD also promotes clock resetting at dusk, a role not described for ELF3.

By studying the clock phase mutant $v v d$, we have gained important insights into the organization of the circadian clockwork under entrainment conditions. VVD helps to establish a functional clock in normal photoperiods by muting the responsiveness of clock components at sunrise and facilitating clock resetting after sunset. As a result, an entrained circadian feedback loop is established that maintains a functional circadian clock throughout the whole LD cycle (Fig. 7D). Without VVD circadian, organization in the light breaks down and so does proper phasing of clock-controlled conidiation (Fig. 7C,E). Given the many similarities already described among circadian systems, it is easy to envision that some of the molecular principles of phasing and entrainment shown here for Neurospora could be conserved across the phylogenetic tree. That is, in addition to wellestablished clock genes, additional components or mechanisms may be discovered that are important for a functional entrained clock but not essential for the operation of the free-running clock.

\section{Materials and methods}

\section{Strains and growth conditions}

All strains used carry the $b d$ mutation to allow for easy assaying of overt rhythmicity on race tubes. General conditions for growth and manipulation are described elsewhere (Davis and deSerres 1970). Strain 289-1 $(\Delta v v d, b d)$ was generated as previously described (Heintzen et al. 2001). Strain 87-3 (vvd $\left.d^{+}, b d\right)$ served as clock wild type. The strain 289-10 ( $\Delta v v d, b d$, his) was used as a parent strain to create the qa-vvd knock-in Neurospora strains (see Constructs). Strain 40-3 (qa-2-vvd, bd) is a representative $q a$ - $v v d$ knock-in strain, originating from a backcross of 289-10 $\mathrm{p}^{\mathrm{pME} 11-2}(q a-2-v v d, b d, \mathrm{~A})$ with 289-1 ( $\left.\Delta v v d, b d, a\right)$ to create homokaryotic progeny. Strains 29-3 $\left(f r q^{10}, b d\right)$ and 29-6 (frq $\left.{ }^{10} \Delta v v d, b d\right)$ originate from a cross between 289-1 $(\Delta v v d, b d, a)$ and 86-1 (frq $\left.{ }^{10}, b d, A\right)$. The genotype of strain 585-7 is $f r q^{7}, b d$, and the genotype of strain 294-4 is $f r q^{7}, v v d^{\mathrm{SS} 692}$.

\section{Race tubes}

All experiments were conducted in light- and temperature-controlled test chambers (Sanyo MLR-350). The light intensities for the LL, LD, skeleton, and light-pulse experiments were all 21 $\mu \mathrm{mol} / \mathrm{m}^{2} / \mathrm{sec}$. For the skeleton photoperiods, complete darkness was interrupted by 15 -min light pulses $\left(21 \mu \mathrm{mol} / \mathrm{m}^{2} / \mathrm{sec}\right)$ separated by 8 - and 16-h intervals, respectively, to mimic dawn and dusk of a short or long day. To mimic short day conditions, cultures were exposed to their first light pulse $8 \mathrm{~h}$ after lights off (following $2 \mathrm{~d}$ of growth in LL), whereas long day conditions were simulated by giving the first light pulse $16 \mathrm{~h}$ into DD. Alternatively, race tube cultures were entrained to full short or long days and then exposed to the corresponding skeleton photoperiods. The release experiment was repeated at light intensities of $21 \mathrm{\mu mol} / \mathrm{m}^{2} / \mathrm{sec}$ and $140 \mathrm{\mu mol} / \mathrm{m}^{2} / \mathrm{sec}(11,000 \mathrm{lux})$ (Supplementary Fig. S1). Race tube media contained $1 \times$ Vogel's salts, $0.1 \%$ glucose, $50 \mathrm{ng} / \mathrm{\mu L}$ biotin, $0.17 \%$ arginine, and $1.5 \%$ agar. For the entrainment experiments, strains were grown in various LD cycles $(4: 20 ; 8: 16 ; 12: 12 ; 16: 8 ; 20: 4)$ over a 3 -d period before being released into constant conditions (either LL or DD) for a further $4 \mathrm{~d}$ at $25^{\circ} \mathrm{C}$. Growth fronts were first marked at both the LD and LL transitions and subsequently every $24 \mathrm{~h}$ in constant conditions. For skeleton photoperiods, strains were kept for $2 \mathrm{~d}$ in LL and either released into 8:16 or 16:8 skeleton photoperiods, released into full photoperiods $(8: 16 ; 16: 8)$, or a combination of $2 \mathrm{~d}$ of full photoperiods and $2 \mathrm{~d}$ of skeleton photoperiods. For light-pulse experiments, race tubes previously kept for at least $24 \mathrm{~h}$ at $25^{\circ} \mathrm{C}$ in LL were transferred to darkness and subsequently subjected to 5 -min light pulses of 21 $\mu \mathrm{mol} / \mathrm{m}^{2} / \mathrm{sec}$ (1750 lux) every $2 \mathrm{~h}$ over a period of $2 \mathrm{~d}$. Phase shifts resulting from light treatment were determined from six replicate controls, and the mean steady-state phase was determined over the next 2-3 d after the light treatment. All race tubes were analyzed using CHRONO (Roenneberg and Taylor 2000).

\section{Liquid culture}

Mycelial mats of strains were grown in petri dishes, and disks were punched using cork borers (size 1) and transferred to Erlenmeyer flasks. Cultures were entrained to $12-\mathrm{h} \mathrm{LD}$ cycles for $2 \mathrm{~d}$ at $25^{\circ} \mathrm{C}$ in $100 \mathrm{~mL}$ of liquid medium consisting of $1 \times$ Vogel's $^{\prime}$ salts, $2 \%$ glucose, $50 \mathrm{ng} / \mu \mathrm{L}$ biotin, and $0.17 \%$ arginine. Subsequently, cultures were transferred to LL at staggered intervals to ensure a similar age at harvest (maximal difference, $8 \mathrm{~h}$ ). In each experiment, disks were no older than $54 \mathrm{~h}$ at the time of harvest.

\section{Constructs}

To make the qa-vvd his3 targeting construct, the $q a-2$ promoter (accession no. X14603) was amplified from Neurospora genomic DNA using the primers 5 '-GGTGGCGGCCGCTAGACTCCT TGACAAGCAGTCG-3' and 5'-CGTGGGTTGGTCTCCTAG GTTTGTGTTAC-3'.

The $v v d$ gene was amplified from a $v v d$ cDNA by using the primers 5'-AGTGGATCCAAACACAATGAGCCATACCGTC AACTCG-3' and 5'-ATCGAATTCATTCCGTTTCGCACTG GAAACC-3'.

PCR products were digested with NotI/BamHI ( $q a-2$ promoter) or BamHI/EcoRI (vvd) and ligated into pBM60 (Margolin et al. 1997) to create pME11. The pME11 plasmid was verified by sequencing from both its $\mathrm{T} 3$ and $\mathrm{T} 7$ sites. This construct was used to transform the parental strain 289-10 ( $\Delta v v d, b d$, his) for targeted integration to the his locus. Putative transformants were selected on minimal medium, analyzed by Southern blot, 
Elvin et al.

and run on race tubes with or without the addition of the inducer quinic acid $\left(10^{-2} \mathrm{M}\right)$.

\section{RNA analyses}

RNA was extracted from liquid cultures and blotted and probed as described previously (Aronson et al. 1994b). For frq detection the plasmid pKAJ104 containing nucleotides 1630-3832 (of the frq ORF) was transcribed into an anti-sense RNA riboprobe, using Amersham ${ }^{32} \mathrm{P}$-dUTP $(800 \mathrm{Ci} / \mathrm{mmol}$ ) to a specific activity of $\sim 109 \mathrm{cpm} / \mu \mathrm{g}$. Similarly, gene-specific riboprobes were obtained for vvd (AF338412, positions 239-1173), al-1 (M57465, positions 1199-2517), and $w C-1$ (X94300, positions 1450-3265) by labeling PCR fragments containing appropriate T7 Polymerase sites to generate anti-sense RNA riboprobes.

\section{Southern blot}

Putative 289-10 $(\Delta v v d, b d)$ transformants that carried the qavvd insertion at the his-3 locus were analyzed by Southern blotting. Genomic DNA was extracted using standard methods, digested with SalI, and separated on a $0.8 \%$ agarose gel. Following blotting to Hybond- $\mathrm{N}^{+}$membrane (Amersham), blots were probed with randomly labeled (Ambion) BglII-PstI fragments of pME11 containing qa-2 promoter, $v v d$ ORF, and his-3 sequences.

\section{Protein extraction and Western blot}

Protein extracts were obtained as previously described (Garceau et al. 1997). One-hundred micrograms of total protein were loaded per lane and, after SDS-PAGE, blotted onto Immobilon-P membrane (Millipore). Membranes were hybridized with antiFRQ and anti-VVD anti-sera, as previously described (Garceau et al. 1997; Heintzen et al. 2001). Western blots were processed according to the manufacturer's instructions (Pierce, Super Signal Kit), and results were visualized on Hyperfilm ECL film (Amersham).

\section{Acknowledgments}

We thank Andrew Millar, Sue Crosthwaite, Nathan PriceLloyd, and Carsten Schwerdtfeger for critical reading of the manuscript and the anonymous reviewers for their helpful suggestions. This work was supported by grants from the National Institute of Health (R37-GM 34985 to J.C.D., MH44651 to J.C.D. and J.J.L.), the National Science Foundation (MCB0084509 to J.J.L.), and the Norris Cotton Cancer Center core grant at Dartmouth Medical School. C.H. was supported by a fellowship of the Swiss National Science Foundation and a NATO fellowship of the German Academic Exchange Service and by a grant from the BBSRC (34/G17511).

\section{References}

Aronson, B.D., Johnson, K.A., and Dunlap, J.C. 1994a. Circadian clock locus frequency: Protein encoded by a single open reading frame defines period length and temperature compensation. Proc. Nat1. Acad. Sci. 91: 7683-7687.

Aronson, B.D., Johnson, K.A., Loros, J.J., and Dunlap, J.C. 1994b. Negative feedback defining a circadian clock: Autoregulation of the clock gene frequency. Science 263: 15781584.

Ashmore, L.J. and Sehgal, A. 2003. A fly's eye view of circadian entrainment. J. Biol. Rhythms 18: 206-216.
Cermakian, N. and Sassone-Corsi, P. 2000. Multilevel regulation of the circadian clock. Nat. Rev. Mol. Cell. Biol. 1: 5967.

Chang, B. and Nakashima, H. 1997. Effects of light-dark cycles on the circadian conidiation rhythm in Neurospora crassa. J. Plant. Res. 110: 449-453.

Cheng, P., He, Q.Y., Yang, Y.H., Wang, L.X., and Liu, Y. 2003. Functional conservation of light, oxygen, or voltage domains in light sensing. Proc. Natl. Acad. Sci. 100: 5938-5943.

Christie, J.M., Swartz, T.E., Bogomolni, R.A., and Briggs, W.R. 2002. Phototropin LOV domains exhibit distinct roles in regulating photoreceptor function. Plant J. 32: 205-219.

Collett, M.A., Dunlap, J.C., and Loros, J.J. 2001. Circadian clock-specific roles for the light response protein WHITE COLLAR-2. Mol. Cell Biol. 21: 2619-2628.

Correa, A., Lewis, A.Z., Greene, A.V., March, I.J., Gomer, R.H., and Bell-Pedersen, D. 2003. Multiple oscillators regulate circadian gene expression in Neurospora. Proc. Nat1. Acad. Sci. 100: 13597-13602.

Covington, M.F., Panda, S., Liu, X.L., Strayer, C.A., Wagner, D.R., and Kay, S.A. 2001. ELF3 modulates resetting of the circadian clock in Arabidopsis. Plant Cell 13: 1305-1315.

Crosthwaite, S.K., Loros, J.J., and Dunlap, J.C. 1995. Light-induced resetting of a circadian clock is mediated by a rapid increase in frequency transcript. Cell 81: 1003-1012.

Davis, R.L. and deSerres, D. 1970. Genetic and microbial research techniques for Neurospora crassa. Meth. Enzymol. 27A: 79-143.

Dharmananda, S. 1980. 'Studies of the circadian clock of Neurospora crassa: Light-induced phase shifting.' Ph.D. thesis, University of California, Santa Cruz.

Dunlap, J.C. 1999. Molecular bases for circadian clocks. Cell 96: $271-290$.

Dunlap, J. and Loros, J. 2004. The Neurospora circadian system. J. Biol. Rhythms 19: 414-424.

Garceau, N.Y., Liu, Y., Loros, J.J., and Dunlap, J.C. 1997. Alternative initiation of translation and time-specific phosphorylation yield multiple forms of the essential clock protein FREQUENCY. Cell 89: 469-476.

Golden, S.S. 2003. Timekeeping in bacteria: The cyanobacterial circadian clock. Curr. Opin. Microbiol. 6: 535-540.

Gooch, V.D. 1985. Effects of light and temperature steps on circadian rhythms of Neurospora crassa and Gonyaulax polyedra. In Temporal order (ed. N.I. Jaeger), pp. 232-237. Springer-Verlag, New York.

Gooch, V.D., Wehseler, R.A., and Gross, C.G. 1994. Temperature effects on the resetting of the phase of the Neurospora circadian rhythm. J. Biol. Rhythms 9: 83-94.

Granshaw, T., Tsukamoto, M., and Brody, S. 2003. Circadian rhythms in Neurospora crassa: Farnesol or geraniol allow expression of rhythmicity in the otherwise arrhythmic strains frq(10), wc-1, and wc-2. I. Biol. Rhythms 18: 287296.

Hastings, M. 2000. Circadian clockwork: Two loops are better than one. Nat. Rev. Neurosci. 1: 143-146.

Heintzen, C., Loros, J.J., and Dunlap, J.C. 2001. The PAS protein VIVID defines a clock-associated feedback loop that represses light input, modulates gating, and regulates clock resetting. Cell 104: 453-464.

Hicks, K.A., Albertson, T.M., and Wagner, D.R. 2001. EARLY FLOWERING3 encodes a novel protein that regulates circadian clock function and flowering in Arabidopsis. Plant Cell 13: $1281-1292$.

Imaizumi, T., Tran, H.G., Swartz, T.E., Briggs, W.R., and Kay, S.A. 2003. FKF1 is essential for photoperiodic-specific light signalling in Arabidopsis. Nature 426: 302-306. 
Johnson, C.H., Elliott, J., Foster, R., Honma, K., and Kronauer, R.E. 2004. Fundamental Properties of circadian rhythms. In Chronobiology (ed. P.J. DeCoursey), pp. 67-107. Sinauer Associates, Inc. Publishers, Sunderland.

Lakin-Thomas, P.L. and Brody, S. 2000. Circadian rhythms in Neurospora crassa: Lipid deficiencies restore robust rhythmicity to null frequency and white-collar mutants. Proc. Natl. Acad. Sci. 97: 256-261.

Loros, J.J. and Feldman, J.F. 1986. Loss of temperature compensation of circadian period length in the frq-9 mutant of Neurospora crassa. J. Biol. Rhythms 1: 187-198.

Margolin, B.S., Freitag, M., and Selker, E.U. 1997. Improved plasmids for gene targeting at the his-3 locus of Neurospora crassa by electroporation. Fungal Genet. Newsl. 44: 34-36.

McWatters, H.G., Bastow, R.M., Hall, A., and Millar, A.J. 2000. The ELF3 zeitnehmer regulates light signalling to the circadian clock. Nature 408: 716-720.

Merrow, M.W., Garceau, N.Y., and Dunlap, J.C. 1997. Dissection of a circadian oscillation into discrete domains. Proc. Natl. Acad. Sci. 94: 3877-3882.

Petersen, E.L. 1980. A limit cycle interpretation of the mosquito circadian oscillator. J. Theor. Biol. 84: 281-310.

Pittendrigh, C. 1981. Circadian systems: Entrainment. In Biological rhythms (ed. J. Aschoff), pp. 95-124. Plenum, New York.

Pregueiro, A.M., Price-Lloyd, N., Bell-Pedersen, D., Heintzen, C., Loros, J.J., and Dunlap, J.C. 2005. Assignment of an essential role for the Neurospora frequency gene in circadian entrainment to temperature cycles. Proc. Natl. Acad. Sci. 102: 2210-2215.

Roenneberg, T. and Taylor, W. 2000. Automated recordings of bioluminescence with special reference to the analysis of circadian rhythms. Methods Enzymol. 305: 104-119.

Roenneberg, T., Daan, S., and Merrow, M. 2003. The art of entrainment. J. Biol. Rhythms 18: 183-194.

Ruoff, P., Vinsjevik, M., Monnerjahn, C., and Rensing, L. 2001. The Goodwin model: Simulating the effect of light pulses on the circadian sporulation rhythm of Neurospora crassa. I. Theor. Biol. 209: 29-42.

Schwerdtfeger, C. and Linden, H. 2001. Blue light adaptation and desensitization of light signal transduction in Neurospora crassa. Mol. Microbiol. 39: 1080-1087.

. 2003. VIVID is a flavoprotein and serves as a fungal blue light photoreceptor for photoadaptation. $E M B O$ J. 22: 4846 4855.

Searle, I. and Coupland, G. 2004. Induction of flowering by seasonal changes in photoperiod. EMBO J. 23: 1217-1222.

Shigeyoshi, Y., Taguchi, K., Yamamoto, S., Takekida, S., Yan, L., Tei, H., Moriya, T., Shibata, S., Loros, J.J., Dunlap, J.C., et al. 1997. Light-induced resetting of a mammalian circadian clock is associated with rapid induction of the mPerl transcript. Cell 91: 1043-1053.

Shrode, L.B., Lewis, Z.A., White, L.D., Bell-Pedersen, D., and Ebbole, D.J. 2001. vvd is required for light adaptation of conidiation-specific genes of Neurospora crassa, but not circadian conidiation. Fungal Genet. Biol. 32: 169-181.

Talora, C., Franchi, L., Linden, H., Ballario, P., and Macino, G. 1999. Role of a white collar-1-white collar-2 complex in blue-light signal transduction. EMBO J. 18: 4961-4968.

Tan, Y., Dragovic, Z., Roenneberg, T., and Merrow, M. 2004a. Entrainment dissociates transcription and translation of a circadian clock gene in Neurospora. Curr. Biol. 14: 433-438.

Tan, Y., Merrow, M., and Roenneberg, T. 2004b. Photoperiodism in Neurospora crassa. J. Biol. Rhythms 19: 135-143.

Tomita, J., Nakajima, M., and Iwasaki, H. 2005. No transcription-translation feedback in circadian rhythm of KaiC phos- phorylation. Science 307: 251-254.

Tyler, B., Geever, R.F., Case, M.E., and Giles, N.H. 1984. Cisacting and trans-acting regulatory mutations define two types of promoters controlled by the qa-1F gene of Neurospora. Cell 36: 493-502.

Yamamoto, Y., Yagita, K., and Okamura, H. 2005. Role of cyclic mPer2 expression in the mammalian cellular clock. Mol. Cell Biol. 25: 1912-1921.

Yanovsky, M.J. and Kay, S.A. 2003. Living by the calendar: How plants know when to flower. Nat. Rev. Mol. Cell. Biol. 4: 265-275.

Young, M. and Kay, S.A. 2001. Timezones: A comparative genetics of circadian clocks. Nat. Rev. Genet. 2: 702-715. 


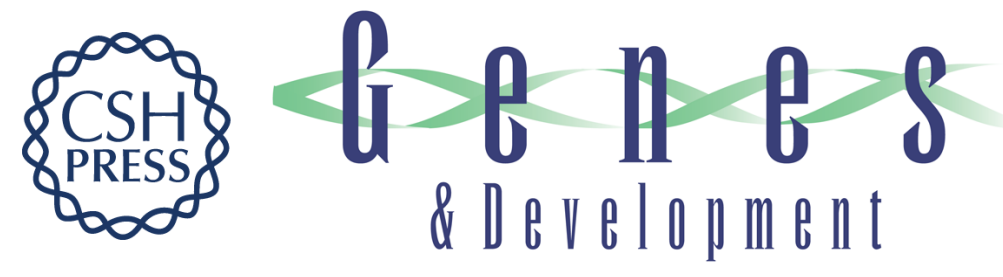

\section{The PAS/LOV protein VIVID supports a rapidly dampened daytime oscillator that facilitates entrainment of the Neurospora circadian clock}

Mark Elvin, Jennifer J. Loros, Jay C. Dunlap, et al.

Genes Dev. 2005, 19:

Access the most recent version at doi:10.1101/gad.349305

Supplemental http://genesdev.cshlp.org/content/suppl/2005/10/20/19.21.2593.DC1

Material

References This article cites 45 articles, 15 of which can be accessed free at:

http://genesdev.cshlp.org/content/19/21/2593.full.html\#ref-list-1

License

Email Alerting Receive free email alerts when new articles cite this article - sign up in the box at the top

Service right corner of the article or click here.

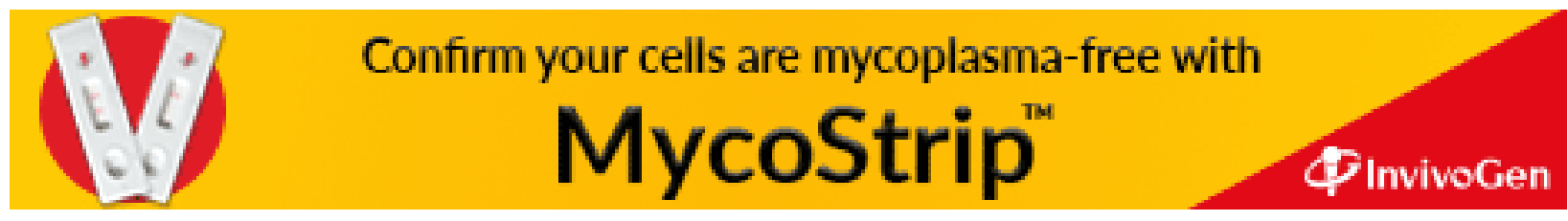

